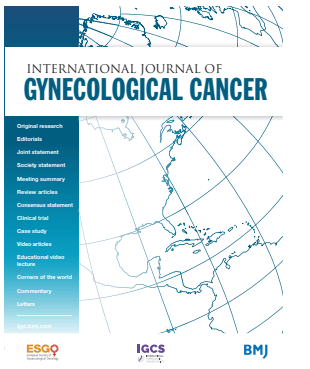

Department of Gynecologic Oncology, La Fe University and Polytechnic Hospital, Valencia, Spain

Correspondence to Dr Pablo Padilla-Iserte, Department of Gynecology Oncolgy, La Fe University and Polytechnic Hospital, Valencia 46026, Spain; pablo_iserte@ hotmail.com

Accepted 16 November 2020 Published Online First 4 December 2020

\title{
Uterine manipulator in endometrial cancer: a video is worth a thousand words
}

Pablo Padilla-Iserte, Raquel Quintana, Tiermes Marina, Victor Lago, Luis Matute, Santiago Domingo

The uterine manipulator is a device commonly used in minimally invasive hysterectomy procedures for benign disease to facilitate uterus mobilization during surgery, generate tension on the main supporting elements of the uterus (broad ligament, uterine vessels, and uterosacral ligaments) to improve surgical field exposure, and provide a landmark for the colpotomy. Nonetheless, the use of a uterine manipulator has not been demonstrated to reduce surgical complications. $^{1}$

With the introduction of minimally invasive approaches in gynecological oncology treatments, this uterine device has been utilized for endometrial and cervical cancers. Without substantial evidence to support its use, surgeons are required to make decisions about using the uterine manipulator in oncological patients based on their surgical experience.

In cervical cancer the LACC (Laparoscopic Approach to Cervical Cancer) trial has shown a worse, unexpected oncological outcome after a minimally invasive surgery approach in early-stage cervical cancer. ${ }^{2}$ One of the hypotheses generated was that the uterine manipulator might influence this worse prognosis. The retrospective SUCCOR trial-an international European cohort observational study comparing minimally invasive surgery versus open abdominal radical hysterectomy in patients with stage IB1 cervical cancer-showed that patients with early stage cervical cancer who underwent minimally invasive surgery using a uterine manipulator had a higher relapse rate than the group undergoing minimally invasive surgery without use of a uterine manipulator, resulting in the same oncological outcomes as patients with open surgery. ${ }^{3}$

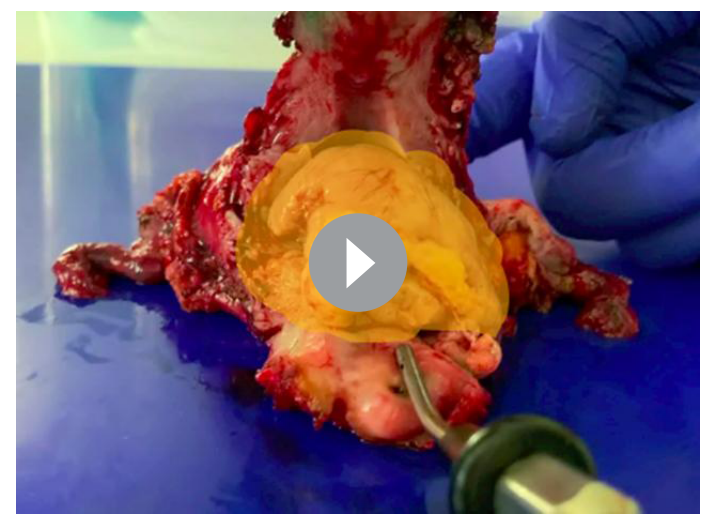

Video 1 Relationship between the uterine manipulator and endometrial tumor.
In endometrial cancer, the current evidence derives from retrospective studies with limited sample sizes. A recent multicenter study evaluating uterine manipulator use in early stage endometrial cancer by minimally invasive surgery found a higher recurrence rate with a worse oncologic outcome in patients with uterus-confined endometrial cancer (International Federation of Gynecology and Obstetrics (FIGO) I-II), which questions the safety of the uterine manipulator in endometrial cancer. ${ }^{4}$

We present a video detailing the interaction between a tumor and the uterine device. The uterine manipulator is inserted vaginally through the cervical canal into a cavity lined with neoplastic tissue. This video displays the macroscopic interaction during uterus mobilization in surgery and its effect on the surgical specimen. The demonstration allows a real, compressive, and visual evaluation of the uterine tumor-uterine manipulator relationship in endometrial cancer.

\section{Twitter Tiermes Marina @Tiermes}

Contributors All authors have actively participated in the elaboration of the manuscript. PP-I: video edition, video locutation, abstract. RQ,TM, VL, LM: designs and review of the video and abstract. SD:abstract, designs and review of the video.

Funding The authors have not declared a specific grant for this research from any funding agency in the public, commercial or not-for-profit sectors.

Competing interests None declared.

Patient consent for publication Not required.

Provenance and peer review Not commissioned; externally peer reviewed.

Data availability statement All data relevant to the study are included in the article.

\section{REFERENCES}

1 van den Haak L, Alleblas C, Nieboer TE, et al. Efficacy and safety of uterine manipulators in laparoscopic surgery: a review. Arch Gynecol Obstet 2015;292:1003-11.

2 Ramirez PT, Frumovitz M, Pareja R, et al. Minimally invasive versus abdominal radical hysterectomy for cervical cancer. N Engl J Med Overseas Ed 2018;379:1895-904.

3 Chiva L, Zanagnolo V, Querleu D, et al. SUCCOR study: an international European cohort observational study comparing minimally invasive surgery versus open abdominal radical hysterectomy in patients with stage IB1 cervical cancer. Int J Gynecol Cancer 2020;30:1269-77.

4 Padilla-Iserte $P$, Lago V, Tauste $C$, et al. Impact of uterine manipulator on oncological outcome in endometrial cancer surgery. Am J Obstet Gynecol 2020;S00029378:30744-4. 